\title{
Estimated intake of dietary phyto-oestrogens in Australian women and evaluation of correlates of phyto-oestrogen intake
}

\author{
Petra H. Lahmann ${ }^{1}$, Maria Celia Hughes ${ }^{1}$, Torukiri I. Ibiebele ${ }^{1}$, Angela A. Mulligan ${ }^{2}$, \\ Gunter G. C. Kuhnle ${ }^{2,3}$ and Penelope M. Webb ${ }^{1}$; Australian Ovarian Cancer Study Management Group \\ and Australian National Endometrial Cancer Study Group \\ ${ }^{1}$ Population Health Department, Queensland Institute of Medical Research, 300 Herston Road, Herston, Brisbane, QLD 4006, Australia \\ ${ }^{2}$ European Prospective Investigation of Cancer, Department of Public Health and Primary Care, University of Cambridge, Strangeways Research \\ Laboratory, Cambridge CB1 8RN, UK \\ ${ }^{3}$ Department of Food and Nutritional Sciences, University of Reading, Reading RG6 6AP, UK
}

(Received 19 December 2011 - Final revision received 14 June 2012 - Accepted 6 July 2012)

Journal of Nutritional Science (2012), vol. 1, e11, page 1 of 9

doi:10.1017/jns.2012.11

\section{Abstract}

The role of dietary phyto-oestrogens in health has been of continued interest and debate, but data available on the distribution of intake in the Australian diet are scarce. Therefore, we aimed to estimate phyto-oestrogen consumption in Australian women, describe the pattern of intake and identify correlates of high phyto-oestrogen intake. Study participants were 2078 control women (18-79 years) from two population-based case-control studies on gynaecological cancers (2002-2007). Dietary information was obtained using a 135-item FFQ, and the intakes of isoflavones, lignans, enterolignans and coumestans, including their individual components, were estimated using a database of phyto-oestrogen content in food developed in the UK. Median total intake (energy-adjusted) of phyto-oestrogens was $1.29 \mathrm{mg} / \mathrm{d}$, including $611 \mu \mathrm{g} / \mathrm{d}$ isoflavones, $639 \mu \mathrm{g} / \mathrm{d}$ lignans, $21 \mu \mathrm{g} / \mathrm{d}$ enterolignans and $8 \mu \mathrm{g} / \mathrm{d}$ coumestrol. Both isoflavone and lignan intakes were strongly skewed towards higher values and positively correlated with age. Women consumed on average two servings of soyabean foods/week. Compared to lower phyto-oestrogen consumers $(\leq 1 \cdot 29 \mathrm{mg} / \mathrm{d}$, median split), higher phyto-oestrogen consumers ( $>1 \cdot 29$ $\mathrm{mg} / \mathrm{d}$ ) were slightly older, less likely to be smokers, had a higher educational and physical activity level, lower BMI, lower intake of dietary fat, and higher intake of fibre, selected micronutrients and soyabean foods (all $P<0.03$ ). The daily intake of phyto-oestrogens in Australian women with predominantly Caucasian ethnicity is approximately $1 \mathrm{mg}$; this is similar to other Western populations, but considerably lower than that among Asian women. However, those with a relatively high phyto-oestrogen diet seem to have a healthier lifestyle and a more favourable dietary profile compared to others.

Key words: Phyto-oestrogens: Isoflavones: Lignans: Coumestans: FFQ

In the recent past, there has been considerable interest in dietary phyto-oestrogens and their potential health benefits or risks ${ }^{(1)}$. Phyto-oestrogens have been suggested to play a role in chronic disease occurrence ${ }^{(2-5)}$, specifically to influence the risk of hormone-sensitive cancers due to their oestrogenic and anti-oestrogenic properties ${ }^{(6-12)}$. Apart from their hormonal activities, phyto-oestrogens have been shown to have antioxidative, antiproliferative, anti-angiogenic and antiinflammatory activities ${ }^{(12,13)}$.

Phyto-oestrogens are widespread polyphenolic plant compounds and can be subdivided into three main classes: isoflavones, lignans and coumestans ${ }^{(2,4,13)}$. Soyabeans and their products, and chick-peas are the predominant source of isoflavones (genistein, daidzein and glycitein), while oilseeds, whole

Abbreviations: ANECS, Australian National Endometrial Cancer Study; AOCS, Australian Ovarian Cancer Study; EPIC, European Investigation into Cancer and Nutrition; LARI, lariciresinol; MAT, matairesinol; PINO, pinoresinol; SECO, secoisolariciresinol.

*Corresponding author: Dr Petra H Lahmann, fax +61 73845 3503, email PLahmann@gmx.de 
grains, legumes, vegetables and berries are common food sources of the more ubiquitous lignans (secoisolariciresinol (SECO), matairesinol (MAT), lariciresinol (LARI) and pinoresinol (PINO)), components of the plant cell wall. Coumestans, of which coumestrol is the main compound consumed by humans, occur predominantly during germination in sprouting legumes. A common feature of many phyto-oestrogens is that their biological activity depends on intestinal bacterial metabolism $^{(6,14)}$. Intestinal gut microflora form the mammalian lignans ('enterolignans'), enterodiol and enterolactone, from inactive plant lignans and possibly other plant precursors. Another metabolite, equol, is produced from the isoflavone daidzein by intestinal bacteria, but this process happens in only about one-third of the population ${ }^{(6,12)}$.

Consumption of phyto-oestrogen-rich foods differs across populations. Soyabean-based foods have long been a staple of Asian diets and the average dietary intake of isoflavones ranges from 5 to $80 \mathrm{mg} / \mathrm{d}^{(12,13,15)}$, whereas in Western populations the reported daily intake is usually $1-3 \mathrm{mg}$ or even less $(1,11-13,16,17)$. Foods commonly consumed in Western societies contain only small amounts of phyto-oestrogens and these largely comprise lignans derived from grain products and beverages. Thus the lignans are usually the most abundant phyto-oestrogens in the Western diet. Differences in the pattern of phytooestrogen intake and associated lifestyle factors may affect the potential benefits from this dietary intake on chronic disease prevention.

In Australia, population-based data on phyto-oestrogen intake are scarce. To date, only two small studies $(n<500)$ have examined dietary intake from isoflavone-rich foods ${ }^{(18)}$ and intakes of isoflavone and lignan phyto-oestrogens ${ }^{(19)}$. The assessment and accurate quantification of intake of total phyto-oestrogens and their components are complex and rely on comprehensive food databases which have not been available until recently.

The aims of our study were to estimate intake of phyto-oestrogens, including major phyto-oestrogen subclasses and components, from food sources among Australian women, by using a comprehensive database from the European Investigation into Cancer and Nutrition (EPIC)-Norfolk, UK. Secondly, we aimed to describe women's pattern of phyto-oestrogen intake and, further, to identify sociodemographic, lifestyle and dietary factors which are correlated with higher phyto-oestrogen intake from food sources.

\section{Methods}

\section{Study population}

Study participants were control women from two populationbased case-control studies conducted in Australia: the Australian Ovarian Cancer Study (AOCS, 2002-5) and the Australian National Endometrial Cancer Study (ANECS, 2005-7). Controls, aged 18-79 years, were randomly selected from the National Electoral Roll (enrolment to vote is compulsory in Australia), and frequency matched to cases with ovarian or endometrial cancer by age (in 5-year groups) and state of residence. Full details of the AOCS and ANECS study recruitment and data collection have been reported previously ${ }^{(20,21)}$.

This study was conducted according to the guidelines laid down in the Declaration of Helsinki and all procedures involving human subjects/patients were approved by the Queensland Institute of Medical Research Human Research Ethics Committee and all relevant hospital and Cancer Registry Human Research Ethics Committees. Written informed consent was obtained from all female subjects/ patients.

Of the 3442 eligible control women contacted in AOCS, $1615(47 \%)$ consented to participate. Women who reported previous bilateral oophorectomy or did not answer the main questionnaire were excluded, leaving 1508 women. Of the 1404 eligible control women with no report on prior hysterectomy contacted in ANECS, 740 (53\%) agreed to participate and completed an interview. For the present study, we additionally excluded ninty-three AOCS and seventy-seven ANECS control women who did not return a FFQ or did not have valid data on energy intake $(\leq 2940 \mathrm{~kJ} / \mathrm{d}$ or $\geq 16800 \mathrm{~kJ} / \mathrm{d}$ ), leaving a final group of 2078 women (1415 AOCS; 663 ANECS) for this analysis. Caucasian ethnicity was reported by $96 \%$ of all women.

\section{Dietary assessment}

Dietary information was obtained using a 135-item semiquantitative FFQ based on the instrument developed by Willett et al. ${ }^{(22)}$, and modified and validated for use in Australia ${ }^{(23-25)}$. Reasonable reproducibility of food and nutrient intake for this FFQ has been documented ${ }^{(26)}$. Women were asked to report on how often they consumed a specified amount of each food item (including items on alcohol consumption) in the previous year.

The FFQ included the following soyabean food items: soyabeans, soyabean milk, soyabean-based meat substitutes and soyabean and linseed bread. For each food, a commonly used unit or portion size was specified, and women were asked to estimate how often, on average, they had eaten the given amount of food over the past year. The nine response options ranged from 'never' to 'four or more times per day.' Women also provided detailed information on the consumption of nutritional supplements.

Average daily intake of phyto-oestrogens was calculated by multiplying the estimated intake of each food item in $\mathrm{g} / \mathrm{d}$ (based on the frequency of consumption and average portion size), by the phyto-oestrogen content of the food using a comprehensive database developed in the $\mathrm{UK}^{(10)}$. This database covered more than $500 \mathrm{UK}$ food items which were analysed for twelve phyto-oestrogens and lignans in triplicate by means of $\mathrm{LC} / \mathrm{MS} / \mathrm{MS}$ and ${ }^{13} \mathrm{C}_{3}$-labelled internal standards as described previously ${ }^{(27,28)}$. Specifically, phyto-oestrogens in foods of animal origin are included in this database ${ }^{(29)}$. For nine food items which were not found in the UK database, a recipe approach was used to estimate phyto-oestrogen intakes. Similar to the $\mathrm{UK}^{(10,30)}$, soyabean is also used in the production of bread in Australia to help improve the bread quality. We compared published data on the isoflavone 
content of bread, soyabean and soyabean products in Australia $^{(31)}$ with the UK database and found the values to be compatible. Plant lignan intake in the UK database was based on SECO and MAT only. The values for LARI and PINO were derived from a published Canadian database ${ }^{(32)}$.

The intakes $(\mu \mathrm{g} / \mathrm{d})$ of the following phyto-oestrogens from food sources were estimated: isoflavones (daidzein, genistein, glycitein, biochanin $\mathrm{A}$ and formononetin), lignans (SECO, MAT, LARI and PINO), enterolignans (enterolactone, enterodiol and equol), coumestrol and total phyto-oestrogens. Equol, a metabolite of daidzein, is not a mammalian lignan, but was included in the subclass of enterolignans because it is formed by intestinal microflora, as is enterodiol and enterolactone ${ }^{(10)}$. Plant lignans are considered to be precursors of their bioactive form and hence precursors of phytooestrogens. In this study, as commonly done by others, we refer to as phyto-oestrogens the group of polyphenolic plant compounds including both active and inactive forms of lignans and isoflavones. The daily intake of energy $(\mathrm{kJ} / \mathrm{d})$, macronutrients $(\mathrm{g} / \mathrm{d})$, vitamins and minerals ( $\mathrm{mg}$ or $\mu \mathrm{g} / \mathrm{d}$ ) was estimated using Australian food composition tables as contained in NUTTAB2006 ${ }^{(33)}$.

\section{Covariates}

Women self-completed a questionnaire (AOCS) or were interviewed in person (ANECS) on health and lifestyle characteristics $^{(20,21)}$ including ethnicity (Caucasian, Asian and others), marital status (single, separated and married), education (high school or less, technical college/diploma, trade certificate/apprenticeship and university), occupation (employed full-time/part-time/retired/unemployed), smoking status (never/ex-/current smoker), alcohol consumption (g/d), recreational physical activity (low, moderate and high level based on the frequency and intensity of activity per week) ${ }^{(34)}$, body size, menopausal status (pre-/peri-/postmenopausal), age at menopause (years), parity (nulliparous/parous), and ever use of oral contraceptives (yes/no) and hormone replacement therapy (yes/no). Women were asked to report their height and weight 1 year before study recruitment. BMI was calculated as weight $(\mathrm{kg})$ divided by height $(\mathrm{m})$ squared, and categorised according to commonly used definitions of overweight and obesity ${ }^{(35)}$.

\section{Statistical analysis}

For all analyses, we used a pooled dataset of participants $(n$ 2078) from both studies AOCS and ANECS. Women participating in AOCS ( $n$ 1415, 19-79 years) did not differ significantly from those participating in ANECS (n 663, 31-79 years) on relevant characteristics, except for age and parity. On average, women from ANECS were older than their counterparts from AOCS (mean age: 61.1 (SD 9.8) v. 56.3 (SD 12.5) years), and were less likely to be nulliparous ( $8 v$. $12 \%$ ). In contrast to AOCS, ANECS by study design included only women who reported no prior hysterectomy.

Intake estimates of dietary phyto-oestrogens were adjusted for total energy intake using the nutrient residual method as described by Willett ${ }^{(22)}$. All dietary variables were logtransformed prior to calculation of the residuals. Women were categorised as lower than median $(\leq 1286 \mu \mathrm{g} / \mathrm{d})$ or higher than median $(>1286 \mu \mathrm{g} / \mathrm{d})$ phyto-oestrogen consumers, based on the median energy-adjusted daily intake of total phyto-oestrogens, and are referred to as 'lower' and 'higher' consumers throughout the article. Daily (weekly) soyabean consumers were defined as women who consumed at least one portion of any of the following foods per d (week): soyabeans, soyabean milk, soyabean-based meat substitutes and soyabean and linseed bread. For lower $v$. higher phyto-oestrogen consumer comparisons, a $\chi^{2}$ test was applied to categorical variables and a Student's $t$ test was conducted for continuous variables. ANOVA was used to determine differences in dietary intake between lower and higher phyto-oestrogen consumers adjusted for age, education, parity, BMI, physical activity and the use of any dietary supplement. We used Spearman correlations to assess the relation between phyto-oestrogen variables and relevant cofactors. Data were analysed using the SAS statistical package (version 9.2; SAS Institute). All statistical tests were twosided, and $P$ values $<0.05$ were considered significant.

Table 1. Daily energy and specific phyto-oestrogen intakes from FFQ in Australian women, 18-79 years ( $n$ 2078)

(Mean values and standard deviations; median values and interquartile ranges)

\begin{tabular}{|c|c|c|c|c|}
\hline & Mean & SD & Median & $\begin{array}{l}\text { Interquartile } \\
\text { range }\end{array}$ \\
\hline Energy (kJ) & 9004 & 2538 & 8763 & 3375 \\
\hline $\begin{array}{l}\text { Percentage of energy } \\
\text { from fat }\end{array}$ & 31.3 & 5.5 & $31 \cdot 2$ & $7 \cdot 0$ \\
\hline $\begin{array}{l}\text { Percentage of energy } \\
\text { from carbohydrates }\end{array}$ & $44 \cdot 3$ & $6 \cdot 3$ & 44.5 & $7 \cdot 7$ \\
\hline $\begin{array}{l}\text { Percentage of energy } \\
\text { from protein }\end{array}$ & $18 \cdot 8$ & 3.0 & $18 \cdot 6$ & 3.9 \\
\hline \multicolumn{5}{|l|}{ Unadjusted intakes $(\mu \mathrm{g})$} \\
\hline Total phyto-oestrogens & 3580.5 & $6755 \cdot 2$ & $1334 \cdot 6$ & 1823.7 \\
\hline Isoflavones & $2826 \cdot 7$ & $6616 \cdot 5$ & 604.0 & $1475 \cdot 6$ \\
\hline Lignans & $718 \cdot 3$ & 370.5 & $644 \cdot 3$ & $390 \cdot 6$ \\
\hline Enterolignans & 23.1 & $14 \cdot 1$ & $20 \cdot 1$ & 17.5 \\
\hline Coumestrol & $12 \cdot 4$ & $18 \cdot 0$ & 7.9 & $7 \cdot 0$ \\
\hline \multicolumn{5}{|l|}{$\begin{array}{l}\text { Energy-adjusted intakes* } \\
\quad(\mu \mathrm{g})\end{array}$} \\
\hline Total phyto-oestrogens & 3293.0 & $5770 \cdot 8$ & $1285 \cdot 8$ & $1693 \cdot 3$ \\
\hline Isoflavones & $2556 \cdot 3$ & 5749.0 & 610.5 & 1488.5 \\
\hline Daidzein & $768 \cdot 3$ & $1717 \cdot 3$ & $197 \cdot 3$ & 452.5 \\
\hline Genistein & 1637.9 & $3822 \cdot 0$ & 344.9 & 953.8 \\
\hline Glycitein & 124.5 & $227 \cdot 3$ & $41 \cdot 1$ & $81 \cdot 0$ \\
\hline Biochanin A & 21.9 & 9.0 & $19 \cdot 7$ & $9 \cdot 1$ \\
\hline Formononetin & 3.7 & 1.8 & $3 \cdot 2$ & 1.6 \\
\hline Lignans & 692.9 & $296 \cdot 4$ & $639 \cdot 0$ & 322.9 \\
\hline Secoisolariciresinol & $474 \cdot 2$ & $243 \cdot 7$ & $426 \cdot 1$ & $232 \cdot 8$ \\
\hline Matairesinol & $68 \cdot 8$ & $36 \cdot 4$ & $58 \cdot 9$ & $41 \cdot 6$ \\
\hline Pinoresinol & $56 \cdot 6$ & $30 \cdot 1$ & $51 \cdot 6$ & $35 \cdot 1$ \\
\hline Lariciresinol & 84.9 & 34.0 & $80 \cdot 3$ & $42 \cdot 8$ \\
\hline Enterolignans & $22 \cdot 3$ & $12 \cdot 4$ & $20 \cdot 6$ & $15 \cdot 1$ \\
\hline Enterolactone & $16 \cdot 5$ & 9.5 & 14.9 & $11 \cdot 3$ \\
\hline Enterodiol & 0.05 & 0.05 & 0.04 & 0.05 \\
\hline Equol & $5 \cdot 8$ & 3.2 & 5.4 & $3 \cdot 7$ \\
\hline Coumestrol & 11.9 & $16 \cdot 0$ & $7 \cdot 8$ & 6.5 \\
\hline
\end{tabular}

*Adjusted using the residual method; nutrients were log-transformed before adjustment. 


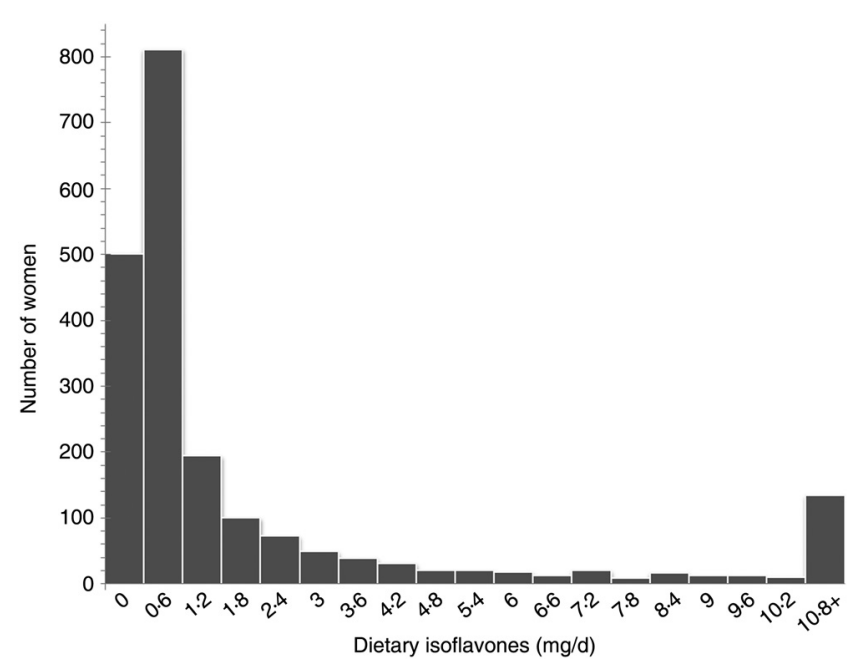

Fig. 1. Distribution of dietary isoflavone intake among 2078 Australian women (2002-2007)

\section{Results}

Women's dietary phyto-oestrogen intake (energy-adjusted) is shown in Table 1. Estimated daily median intake of total phyto-oestrogens was $1.29 \mathrm{mg}$ (mean 3.4 (sD 5.8) $\mathrm{mg}$ ) of which $0.6 \mathrm{mg}$ was derived from isoflavones and $0.6 \mathrm{mg}$ from lignans. As expected, enterolignans (median intake 21 $\mu \mathrm{g} / \mathrm{d}$ ) and coumestrol (median intake $8 \mu \mathrm{g} / \mathrm{d}$ ) were consumed in very small amounts. Isoflavone intake ranged from 45 to 71 $908 \mu \mathrm{g} / \mathrm{d}$ and was strongly skewed towards higher values (Fig. 1), as was lignan intake (range: 101-2515 $\mu \mathrm{g} / \mathrm{d}$ ), although to a lesser extent (Fig. 2). For the total study sample, genistein and daidzein contributed most to the isoflavone intake $(88 \%)$, SECO (66\%) most to lignan intake, while enterolactone $(73 \%)$ was the predominant enterolignan.

Of all women, $11 \%(30 \%)$ were daily (weekly) soyabean food consumers, $59 \%$ used dietary supplements and $21 \%$ used phyto-oestrogen-containing supplements regularly. On average, women consumed two servings of soyabean foods/ week of which soyabean/linseed bread (1.2 servings/week)

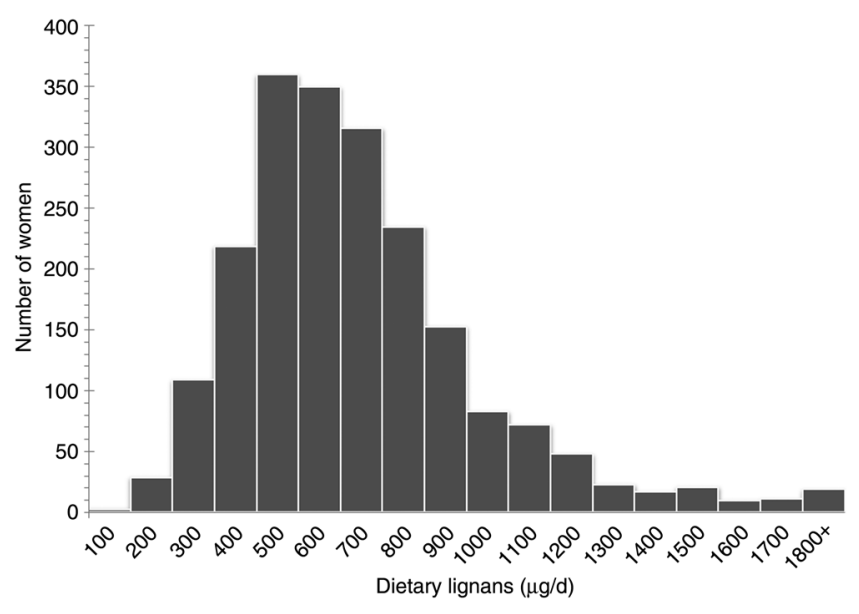

Fig. 2. Distribution of dietary lignan intake among 2078 Australian women (2002-2007). was the dominant soyabean item, followed by soyabean milk products $(0.7$ servings/week). Total phyto-oestrogen intake was positively correlated with age $(r 0 \cdot 07)$ and negatively correlated with BMI $(r-0 \cdot 08)$ (both $P<0 \cdot 001)$. Specifically, lignan intake $(r 0 \cdot 18, P<0 \cdot 0001)$ was more strongly correlated with age than isoflavone intake $(r 0 \cdot 04, P=0 \cdot 06)$.

Characteristics for women grouped as lower or higher dietary phyto-oestrogen consumers are presented in Table 2. Higher phyto-oestrogen consumers were slightly older, more likely to have a higher education and leisure activity level, to be nulliparous, dietary and phyto-oestrogen supplement users and daily soyabean food consumers compared to their counterparts with lower phyto-oestrogen intake. Furthermore, higher phyto-oestrogen consumers tended to have a lower mean body weight and BMI and accordingly were less likely to be overweight or obese than lower phytooestrogen consumers. They were also less likely to be smokers. Other characteristics were not significantly associated with the level of phyto-oestrogen intake.

Table 3 gives estimates of daily energy, nutrient and phytooestrogen intake in higher and lower consumers adjusted for various factors shown to be associated with phyto-oestrogen intake. On average, women who were defined as lower consumers had a median phyto-oestrogen intake of 0.9 (interquartile range 0.3$) \mathrm{mg} / \mathrm{d}$ from diet, whereas higher consumers had a total median intake of 2.6 (interquartile range 4.6 ) $\mathrm{mg} / \mathrm{d}$. Of the higher-intake group, $22 \%$ consumed soyabean food on a daily basis ( $0 \%$ in the lower-intake group). The difference in phyto-oestrogen consumption was therefore especially large for isoflavones (median intake $1.8 v .0 .3 \mathrm{mg} / \mathrm{d}$ ), and less marked for the other three subclasses. Except for protein, $\mathrm{Ca}, \mathrm{Zn}$ and alcohol, intakes of all nutrients were significantly different between the lower- and higher-intake groups. Overall, energy-adjusted mean intakes of carbohydrates, fibre, vitamins and minerals were higher and those of total and saturated fat were lower in women with a higher phytooestrogen intake, indicating an overall healthier dietary profile.

The more favourable dietary pattern was also reflected in other eating behaviours assessed by short questions in the FFQ (data not shown). Higher consumers were more likely to consume $\geq 2$ servings of fruit $(70 \%), \geq 5$ servings of vegetables $(15 \%)$, or $\geq 4$ servings of cereals $(35 \%)$ per $\mathrm{d}$ compared to women with lower phyto-oestrogen intake $(57,8.5$ and $24 \%$ ).

Bread was the major food source of dietary phyto-oestrogens in both lower and higher consumers, but this differed by type for the two groups as indicated in Table 4. In higher consumers soyabean/linseed bread, soyabean milk and nonsoyabean bread contributed each $12 \%$ or more to the daily intake of phyto-oestrogens, while non-soyabean bread, vegetables (other than soyabeans/legumes) and fruit were the predominant sources (each $>16 \%$ ) of phyto-oestrogens among lower consumers. Among lower consumers, beverages, other than soyabean milk, provided a moderate contribution to daily phyto-oestrogen intake, specifically to intakes of lignans (coffee, tea and wine) and enterolignans (non-soyabean milk). In a sub-analysis (data not shown), we examined the contribution of phyto-oestrogen-containing supplements to 
Table 2. Demographic, lifestyle and reproductive characteristics of Australian women by level of total dietary phyto-oestrogen intake ( $n$ 2078) (Percentages or mean values and standard deviations)

\begin{tabular}{|c|c|c|c|}
\hline \multirow[b]{2}{*}{ Variables } & \multicolumn{2}{|c|}{ Total phyto-oestrogen intake } & \multirow[b]{2}{*}{$P$} \\
\hline & 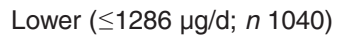 & 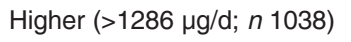 & \\
\hline \multicolumn{4}{|l|}{ Age, categorical (\%) } \\
\hline$<45$ years & $16 \cdot 5$ & $12 \cdot 4$ & 0.03 \\
\hline $45-59$ years & 39.5 & $40 \cdot 8$ & \\
\hline$\geq 60$ years & $44 \cdot 0$ & $46 \cdot 8$ & \\
\hline \multicolumn{4}{|l|}{ Marital status (\%) } \\
\hline Single/never married & $5 \cdot 3$ & $6 \cdot 8$ & 0.30 \\
\hline Separated & $18 \cdot 6$ & $19 \cdot 1$ & \\
\hline Married/de facto & $76 \cdot 1$ & $74 \cdot 1$ & \\
\hline \multicolumn{4}{|l|}{ Menopausal status (\%) } \\
\hline Pre-menopausal & $25 \cdot 3$ & $20 \cdot 3$ & 0.02 \\
\hline Peri-menopausal & $5 \cdot 8$ & $7 \cdot 1$ & \\
\hline Post-menopausal & 68.9 & $72 \cdot 6$ & \\
\hline Age at menopause (years) & & & 0.40 \\
\hline Mean & $49 \cdot 9$ & $50 \cdot 1$ & \\
\hline SD & 4.6 & 4.5 & \\
\hline \multicolumn{4}{|l|}{ Ethnicity (\%) } \\
\hline Caucasian & $96 \cdot 2$ & 95.5 & 0.61 \\
\hline Asian & 1.6 & $2 \cdot 1$ & \\
\hline Others & $2 \cdot 2$ & $2 \cdot 3$ & \\
\hline Education after high school (\%) & $47 \cdot 3$ & $55 \cdot 1$ & 0.0004 \\
\hline \multicolumn{4}{|l|}{ Employment (\%) } \\
\hline Full-time & $24 \cdot 0$ & $22 \cdot 4$ & 0.25 \\
\hline Part-time & 24.4 & $24 \cdot 7$ & \\
\hline Retired & $28 \cdot 1$ & $31 \cdot 6$ & \\
\hline Unemployed & $23 \cdot 6$ & $21 \cdot 2$ & \\
\hline Nulliparous (\%) & $8 \cdot 7$ & $12 \cdot 3$ & 0.06 \\
\hline Ever use of oral contraceptives (\%) & $80 \cdot 2$ & 79.9 & 0.88 \\
\hline Ever use of systemic hormone replacement therapy (\%) & $33 \cdot 3$ & $36 \cdot 9$ & 0.09 \\
\hline Weight (kg) & & & $<0.0001$ \\
\hline Mean & 71.8 & $68 \cdot 8$ & \\
\hline SD & $16 \cdot 6$ & $14 \cdot 2$ & \\
\hline Height (cm) & & & 0.95 \\
\hline Mean & $162 \cdot 7$ & $162 \cdot 7$ & \\
\hline SD & 6.7 & $6 \cdot 8$ & \\
\hline \multicolumn{4}{|l|}{ BMI, categorical (\%) } \\
\hline$<25.0 \mathrm{~kg} / \mathrm{m}^{2}$ & $42 \cdot 4$ & $50 \cdot 9$ & 0.0004 \\
\hline $25.0-29.9 \mathrm{~kg} / \mathrm{m}^{2}$ & 32.5 & $29 \cdot 1$ & \\
\hline$\geq 30 \mathrm{~kg} / \mathrm{m}^{2}$ & $25 \cdot 1$ & $20 \cdot 0$ & \\
\hline \multicolumn{4}{|l|}{ Smoking status (\%) } \\
\hline Never & $60 \cdot 4$ & $58 \cdot 0$ & 0.02 \\
\hline Past smoker & 27.5 & $32 \cdot 6$ & \\
\hline Current smoker & $12 \cdot 1$ & $9 \cdot 5$ & \\
\hline \multicolumn{4}{|l|}{ Leisure physical activity (\%) } \\
\hline Low & $20 \cdot 2$ & $13 \cdot 4$ & $<0.0001$ \\
\hline Medium & 39.9 & $38 \cdot 2$ & \\
\hline High & 39.9 & $48 \cdot 4$ & \\
\hline Daily soya consumer (\%) & 0.0 & 21.9 & $<0.0001$ \\
\hline Use of any dietary supplement (\%) & $53 \cdot 3$ & $64 \cdot 7$ & $<0.0001$ \\
\hline Use of phyto-oestrogen supplement (\%) & $17 \cdot 9$ & $24 \cdot 3$ & 0.0003 \\
\hline
\end{tabular}

total phyto-oestrogen intake, including phyto-oestrogens derived from food sources and supplements. Among users $(21 \%)$ of phyto-oestrogen-containing supplements, supplements contributed only 0.06 (interquartile range 0.17 ) $\%$ to the total daily intake of phyto-oestrogens.

\section{Discussion}

To our knowledge, this investigation is the first to report on the three main classes of dietary phyto-oestrogens in Australian women: isoflavones, lignans (and separately enterolignans) and coumestans, including their components. Two previous much smaller $(n<476)$ Australian studies examined isoflavone-rich foods ${ }^{(18)}$, or total isoflavone and lignan intakes ${ }^{(19)}$ only. In this cohort of 2078 women, aged 18-79 years, who participated as controls in two major population-based case-control studies on gynaecological cancers between 2002 and 2007, we observed that median intake of total phyto-oestrogens (energy-adjusted) from dietary sources was slightly over $1 \mathrm{mg} / \mathrm{d}$ (1286 (interquartile range 1693) $\mu \mathrm{g} / \mathrm{d}$ ). Dietary isoflavone intake (median 611 $\mu \mathrm{g} / \mathrm{d}$ ) was similar to lignan intake (median $639 \mu \mathrm{g} / \mathrm{d}$ ), while the intake of enterolignans (median $21 \mu \mathrm{g} / \mathrm{d}$ ) and coumestrol 
Table 3. Daily energy and energy-adjusted intakes of selected nutrients and main classes of phyto-oestrogens of Australian women by level of total dietary phyto-oestrogen intake ( $n$ 2078) $†$

(Mean values and standard deviations; median values and interquartile ranges)

\begin{tabular}{|c|c|c|c|c|c|c|c|c|}
\hline \multirow[b]{2}{*}{ Nutrient } & \multicolumn{4}{|c|}{ Lower $(\leq 1286$ g/d; $n$ 1040) } & \multicolumn{4}{|c|}{ Higher (>1286 $\mu \mathrm{g} / \mathrm{d} ; n$ 1038) } \\
\hline & Mean & SD & Median & Interquartile range & Mean & SD & Median & Interquartile range \\
\hline Energy $(\mathrm{kJ})$ & $9110^{*}$ & 2503 & 8892 & 3330 & 8897 & 2570 & 8643 & 3445 \\
\hline Percentage of energy from fat & $32 \cdot 3^{*}$ & 5.4 & $32 \cdot 1$ & 6.7 & $30 \cdot 3$ & 5.5 & $30 \cdot 1$ & 6.8 \\
\hline Percentage of energy from carbohydrate & $44 \cdot 0$ & $6 \cdot 3$ & $44 \cdot 0$ & $7 \cdot 7$ & 44.6 & $6 \cdot 2$ & $45 \cdot 1$ & $7 \cdot 7$ \\
\hline Percentage of energy from protein & $18 \cdot 8$ & $3 \cdot 1$ & 18.5 & 3.8 & $18 \cdot 8$ & $3 \cdot 1$ & $18 \cdot 8$ & 3.9 \\
\hline \multicolumn{9}{|l|}{ Energy-adjusted intakes $\ddagger$} \\
\hline Total phyto-estrogens $(\mu \mathrm{g})$ & $923.4^{*}$ & 224.5 & $937 \cdot 4$ & $346 \cdot 1$ & 5667.6 & 7440.5 & 2631.5 & $4591 \cdot 0$ \\
\hline Isoflavones§ $(\mu \mathrm{g})$ & $349 \cdot 2^{*}$ & $175 \cdot 1$ & 315.5 & 252.4 & 4767.6 & $7509 \cdot 2$ & $1799 \cdot 6$ & 4248.6 \\
\hline Lignans $\|(\mu \mathrm{g})$ & $541 \cdot 3^{*}$ & 171.5 & 528.4 & $224 \cdot 9$ & 8447 & $316 \cdot 8$ & 775.6 & 351.0 \\
\hline Enterolignans $\|(\mu \mathrm{g})$ & $23.4^{*}$ & 12.9 & $21 \cdot 0$ & $15 \cdot 7$ & $21 \cdot 2$ & 11.8 & $20 \cdot 1$ & $14 \cdot 8$ \\
\hline Coumestrol $(\mu \mathrm{g})$ & $9 \cdot 3^{*}$ & 9.5 & 6.9 & $5 \cdot 6$ & $14 \cdot 2$ & $20 \cdot 2$ & $9 \cdot 1$ & $7 \cdot 7$ \\
\hline Protein $(\mathrm{g})$ & 96.9 & $15 \cdot 6$ & $95 \cdot 8$ & 19.5 & 97.1 & $15 \cdot 2$ & $96 \cdot 2$ & 19.4 \\
\hline Carbohydrate (g) & $227 \cdot 2^{*}$ & $32 \cdot 3$ & 227.7 & 49.8 & $230 \cdot 2$ & $32 \cdot 3$ & 230.6 & $40 \cdot 1$ \\
\hline Fat $(\mathrm{g})$ & $74 \cdot 1^{*}$ & $12 \cdot 3$ & 73.5 & $15 \cdot 7$ & 69.5 & $12 \cdot 6$ & $69 \cdot 3$ & $15 \cdot 5$ \\
\hline Saturated fat $(\mathrm{g})$ & $28.5^{*}$ & 6.7 & $27 \cdot 8$ & 8.5 & 24.5 & 6.4 & 23.9 & 7.9 \\
\hline Monounsaturated fat (g) & $26 \cdot 7^{*}$ & $5 \cdot 3$ & $26 \cdot 3$ & $6 \cdot 3$ & 25.4 & 5.6 & $25 \cdot 4$ & $7 \cdot 1$ \\
\hline Polyunsaturated fat (g) & $11 \cdot 7^{*}$ & $2 \cdot 7$ & $11 \cdot 2$ & 3.5 & $12 \cdot 3$ & $3 \cdot 2$ & 11.9 & 3.9 \\
\hline Cholesterol (mg) & $268 \cdot 7^{\star}$ & 88.5 & 256.9 & $90 \cdot 2$ & 242.8 & $79 \cdot 2$ & $236 \cdot 0$ & 89.4 \\
\hline Fibre (g) & $30 \cdot 2^{*}$ & 7.4 & 29.8 & 9.4 & 35.7 & 8.4 & 34.9 & $10 \cdot 4$ \\
\hline Alcohol (g) & 6.5 & 9.4 & $2 \cdot 4$ & $9 \cdot 3$ & $8 \cdot 2$ & 11.4 & 3.6 & $10 \cdot 7$ \\
\hline Total folate $(\mu \mathrm{g})$ & $374.5^{\star}$ & $115 \cdot 5$ & $361 \cdot 3$ & 143.9 & 401.0 & $122 \cdot 8$ & $385 \cdot 0$ & 143.6 \\
\hline Vitamin C (mg) & $173.5^{\star}$ & 71.6 & 161.9 & 85.9 & $197 \cdot 7$ & $78 \cdot 3$ & $187 \cdot 7$ & 93.9 \\
\hline$\beta$-Carotene $(\mu \mathrm{g})$ & $5388.0^{*}$ & $2422 \cdot 3$ & 5075.5 & 3154.7 & $6547 \cdot 6$ & 3292.5 & $5966 \cdot 4$ & $3610 \cdot 2$ \\
\hline Vitamin E (mg) & $7 \cdot 6^{*}$ & $2 \cdot 0$ & 7.4 & $2 \cdot 7$ & $8 \cdot 2$ & $2 \cdot 2$ & 7.9 & 2.5 \\
\hline $\mathrm{Ca}(\mathrm{mg})$ & $995 \cdot 2$ & $349 \cdot 1$ & 937.7 & 422.5 & $1017 \cdot 5$ & $323 \cdot 8$ & $982 \cdot 3$ & 404.4 \\
\hline $\mathrm{Fe}(\mathrm{mg})$ & $12 \cdot 7^{*}$ & $2 \cdot 2$ & $12 \cdot 6$ & $2 \cdot 6$ & 13.9 & $2 \cdot 4$ & $13 \cdot 8$ & 2.9 \\
\hline$M g(m g)$ & $360 \cdot 7^{*}$ & 54.4 & 360.9 & 72.5 & $406 \cdot 1$ & $55 \cdot 1$ & $402 \cdot 2$ & 69.9 \\
\hline $\mathrm{Zn}(\mathrm{mg})$ & $13 \cdot 3$ & $2 \cdot 2$ & 13.1 & $2 \cdot 7$ & 13.0 & $2 \cdot 2$ & 12.9 & 2.9 \\
\hline
\end{tabular}

${ }^{*}$ Mean value was significantly different from that of the higher consumers $(P<0.05)$.

† Adjusted for age, education, parity, BMI, physical activity and use of any dietary supplement.

$\ddagger$ Adjusted using the residual method; nutrients were log-transformed before adjustment.

§ Including daidzein, genistein, glycitein, biochanin A and formononetin

II Including secoisolariciresinol, matairesinol, lariciresinol and pinoresinol.

ๆ Including enterolactone, enterodiol and equol.

(median $8 \mu \mathrm{g} / \mathrm{d}$ ) was low. Soyabean foods were the predominant source of phyto-oestrogens. Overall, women consumed on average two servings of soyabean foods per week, whereas among higher phyto-oestrogen consumers, $22 \%$ ate soyabean foods on a daily basis. Women who were grouped as higher phyto-oestrogen consumers were somewhat older, had a higher

Table 4. Consumption and percentage contribution of food groups to total daily intake of phyto-oestrogens (PE) from food sources in Australian women grouped under lower and higher PE consumers

\begin{tabular}{|c|c|c|c|c|}
\hline \multirow[b]{2}{*}{ Food groups } & \multicolumn{2}{|c|}{ Lower consumers ( $\leq 1286 \mu \mathrm{g} / \mathrm{d} ; n$ 1040) } & \multicolumn{2}{|c|}{ Higher consumers $(>1286 \mu \mathrm{g} / \mathrm{d} ; n$ 1038) } \\
\hline & $g / d$ & Percentage of total $\mathrm{PE}^{*}$ & $g / d$ & Percentage of total PE* \\
\hline Soyabean and linseed bread & 0.3 & 3.0 & $9 \cdot 3$ & $21 \cdot 8$ \\
\hline Soyabean milk & 0.07 & 0.3 & $51 \cdot 1$ & 18.6 \\
\hline Non-soyabean bread & $46 \cdot 6$ & 23.2 & $50 \cdot 9$ & 11.9 \\
\hline Vegetables not including potatoes, soyabeans and other legumes & 285.3 & 22.5 & 335.6 & $10 \cdot 6$ \\
\hline Fruits & 515.6 & $16 \cdot 1$ & 597.4 & $8 \cdot 0$ \\
\hline Soyabeans & 0.05 & 0.7 & $2 \cdot 6$ & $6 \cdot 3$ \\
\hline Breakfast cereals & $56 \cdot 4$ & $1 \cdot 3$ & $60 \cdot 2$ & 4.9 \\
\hline Soyabean-based meat substitutes & 0.09 & 0 & $3 \cdot 8$ & 3.8 \\
\hline Coffee & $365 \cdot 0$ & $7 \cdot 1$ & $426 \cdot 3$ & 3.6 \\
\hline Tea & 461.6 & $6 \cdot 0$ & $526 \cdot 2$ & $2 \cdot 6$ \\
\hline Non-soyabean milk & 315.9 & 4.7 & 248.4 & 1.6 \\
\hline Legumes, not including soyabeans & $59 \cdot 2$ & 3.3 & $63 \cdot 1$ & 1.6 \\
\hline Wines & $51 \cdot 6$ & $2 \cdot 4$ & 73.5 & 1.5 \\
\hline Nuts & 5.4 & 1.8 & 8.2 & 0.9 \\
\hline Other dairy products & $126 \cdot 8$ & $2 \cdot 3$ & 124.9 & 0.8 \\
\hline Meat, chicken, seafood and eggs & $220 \cdot 3$ & 1.7 & 198.6 & 0.5 \\
\hline All other foods $\dagger$ & 1138.9 & 3.6 & $1092 \cdot 3$ & 1.0 \\
\hline
\end{tabular}

* Ordered by percentage contribution in higher consumers (total PE $>1286 \mu \mathrm{g} / \mathrm{d}$ ).

$\dagger$ Includes foods contributing $<0.5 \%$ among higher consumers. 
educational level, healthier lifestyle and more favourable dietary profile compared to the rest of the cohort.

Overall, the isoflavone intake in our study is comparable to other reports from Western female populations ${ }^{(11-13,16,17,36-40)}$ with average isoflavone consumption of $\leq 1$ to $2 \mathrm{mg} / \mathrm{d}$. This is considerably lower than the level found in typical Asian soyabean-based diets ${ }^{(12,13,15,18,41)}$ and also below levels where physiological effects are expected (45-100 $\mathrm{mg} / \mathrm{d})^{(1,17,42)}$. Notably, the median dietary isoflavone intake was relatively high when compared to that reported $(30 \mu \mathrm{g} / \mathrm{d})$ in the Australian Longitudinal Assessment of Ageing in Women study ( $n 475,40-80$ years $)^{(19)}$, and more similar to data from UK women (mean 979 (SD 1859) $\mu \mathrm{g} / \mathrm{d}$ ) participating in the EPIC-Norfolk study that used the same phyto-oestrogen database as the current analysis ${ }^{(11)}$. The low daily median intake of dietary isoflavones in the Longitudinal Assessment of Ageing in Women study is unexpected given that this estimate is based on a 112-item FFQ including thirty soyabean foods, and therefore one would expect to see a greater estimated intake of isoflavones compared to the FFQ in the present investigation which contained only five soyabean foods.

In contrast, in the present study, dietary lignan intake was about $35 \%$ of the amount reported (median $1830 \mu \mathrm{g} / \mathrm{d}$ ) for women in the Australian Longitudinal Assessment of Ageing in Women study ${ }^{(19)}$. Lignan values from that study were based on published values for enterolactone and enterodiol, assessed by an indirect in vitro fermentation assay and thus include all sources of plant lignans ${ }^{(19,43)}$. Differences in average intakes may be due to the use of these different assessment methods and databases. When comparing our data with earlier studies of women in Europe and North America which based total lignan intake on either two compounds (SECO and MAT) ${ }^{(11,13,16,37)}$ or on four compounds (SECO, MAT, LARI and PINO $)^{(38,44,45)}$ or more ${ }^{(36)}$, average consumption of plant lignans was compatible and varied between 177 and $1100 \mu \mathrm{g} / \mathrm{d}$ (median). Lignan intake increased with age, which is in accordance with the findings in Dutch women ${ }^{(45)}$, but in contrast to other recent Australian data showing no variation by age $e^{(19)}$.

Women's estimated mean (or median) intake of enterolignans derived from foods of animal origin was similar to levels reported for women (control women for breast cancer cases) in the EPIC-Norfolk study (enterolactone and equol) and women (non-cases) in a Swedish breast cancer study (enterolactone, enterodiol and equol) ${ }^{(8,10)}$. The median intake of coumestrol $(8 \mu \mathrm{g} / \mathrm{d})$ in our study was slightly higher than the reported values from other Western countries (all $\leq 2$ $\mu \mathrm{g} / \mathrm{d})^{(8,13,16,36,38,40,44)}$ except for one ${ }^{(39)}$ reporting a mean intake of coumestrol of $114 \mu \mathrm{g} / \mathrm{d}$ for US women participating in a prospective breast cancer study.

In contrast to some other Western female populations ${ }^{(8,13,16,38,40)}$, the average isoflavone intake in this cohort of women was also high and similar to lignan intake and this is probably due to the relatively high consumption of soyabean foods and the soyabean added to bread to improve the bread quality. One-third of all women reported consumption of at least one serving of soyabean food per week as estimated from the FFQ data; among higher phyto-oestrogen consumers this proportion was almost twice as high (58\%). This compares to $50 \%$ consuming $\geq 1$ serving per week among Australian-born women (n 390, 51-62 years) participating in the population-based Melbourne Women's Midlife Health study ${ }^{(18)}$ and $40 \%$ consuming $\geq 1$ serving during the past month in the Longitudinal Assessment of Ageing in Women study ${ }^{(19)}$. A common feature of these Australian female cohorts, including ours, is that the most frequently consumed soyabean food is soyabean/linseed bread with a reported prevalence of $30-38 \%{ }^{(18,19)}$, and $44 \%$ in the present study. By contrast, among European women ${ }^{(46)}$ soyabean dairy substitutes of which $75 \%$ were liquid items were consumed the most frequently of the seven soyabean product groups examined. Similarly, other studies from Europe and North America have identified soyabean products other than bread as the main soyabean sources for women ${ }^{(38,47-49)}$.

Higher phyto-oestrogen consumers differed significantly from women who consumed lower levels in other dietary, socio-demographic and lifestyle factors. In the present study, increased phyto-oestrogen consumption was associated with higher education and a healthier lifestyle indicated by lower smoking prevalence, lower BMI and higher physical activity level. This is consistent with previous evidence from some ${ }^{(18,19,45)}$, but not all ${ }^{(48)}$ cross-sectional studies. In the latter small study of postmenopausal US women ( $n$ 96), plasma isoflavone concentrations were not associated with education, BMI or activity level. As expected, higher phyto-oestrogen intake was indicative of a beneficial dietary profile, with percentage of energy from fat and fibre closer to current dietary guidelines and higher intakes of selected vitamins and minerals from food sources, and this finding is compatible with other studies ${ }^{(19,45,48)}$. The proportion of users of phyto-oestrogencontaining supplements was slightly higher among higher phyto-oestrogen consumers compared to lower phyto-oestrogen consumers $(24 v .18 \%)$. However, the contribution from these supplements to the total daily intake of phyto-oestrogens (diet and supplement) among users was negligible $(<1 \%)$. Although the cross-sectional design does not provide information on causal (temporal) relationships, our findings may suggest a possible association between dietary phyto-oestrogen intake and health-related behaviours.

Some limitations of our study warrant consideration. The participation rate among controls was relatively low in AOCS (47\%) and ANECS (53\%); hence these women may not be representative of the Australian female population. However, previous comparisons of the AOCS controls to the 2004 Australian National Health Survey, a representative survey of the Australian adult population ${ }^{(50)}$, have shown that the distributions of key variables (education level, parity, BMI and ever/never smoking status) were comparable with national data ${ }^{(21,51)}$

The 135-item FFQ used in this study included the most commonly consumed soyabean-food items in the Australian diet, but was not designed to specifically investigate intake of phyto-oestrogens or their respective subclasses. As a result, consumption of selected foods, such as seeds (important for lignan intake), was not queried in detail and may have been underestimated. However, the FFQ was designed to reflect 
dietary intake of Australians, with food groups such as grain products, fruits and vegetables, which are usually rich in lignans, being well captured ${ }^{(23-25)}$. Establishing the phyto-oestrogen content in foods and quantifying dietary isoflavones and lignans has been challenging and has led to the compilation of several databases over the last two decades. In this study, we used a recently developed comprehensive UK database which allowed for more accurate estimation of phyto-oestrogen exposure, including the phyto-oestrogen content of foods of animal origin ${ }^{(10)}$. Recently, the lignan compounds LARI and PINO were shown to contribute a higher proportion to the overall lignan intake than SECO and $\mathrm{MAT}^{(45)}$. However, in our study, the inclusion of these two compounds did not substantially increase the total lignan intake, and SECO remained the major contributing component of plant lignans.

In this cohort, $4 \%$ of women were of Asian or other non-Caucasian ethnicity, including Indigenous people. As expected, their average phyto-oestrogen intake was somewhat higher than that observed for the majority of the cohort, mainly attributed to increased isoflavone intake (data not shown). However, ethnicity was not a correlate of phyto-oestrogen intake when comparing lower and higher consumers.

In summary, in this population-based cohort of predominantly middle-aged and older women, the largest Australian investigation on this topic to date, dietary phyto-oestrogen intake was similar to levels reported by other Western population studies, and substantially lower than that published for Asian women. Among the subclasses examined, isoflavones contributed most to the total phyto-oestrogen intake, and similar to other Australian data soyabean/linseed bread and other bread were the predominant food source of isoflavones and plant lignans. This study suggests that dietary phyto-oestrogen intake, specifically that of plant lignans, is correlated with older age in women whose age ranged from 18 to 79 years. This association may reflect a perception that these compounds are associated with health benefits, such as alleviation of menopausal symptoms ${ }^{(1)}$, or alternatively a healthier lifestyle with ageing. Interestingly, women in the present study who used phyto-oestrogen-containing supplements $(21 \%)$ were on average slightly younger (mean age $57.8 v .59 .0$ years) and more likely to be pre- or peri-menopausal compared to those not using this type of supplement. This is likely to reflect the use of supplements during early menopausal transition.

Overall, in this female population, higher phyto-oestrogen consumption was correlated with higher dietary fibre and lower fat intake and characteristics indicative of a healthier lifestyle such as non-smoking, lower BMI and higher recreational physical activity level. These factors should be considered as potential confounding variables or effect modifiers in future epidemiological research where much of the evidence to date remains inconclusive regarding the role of dietary phyto-oestrogens in women's health.

\section{Acknowledgements}

The authors declare that they have no conflict of interest. The AOCS was supported by the US Army Medical Research and
Materiel Command under DAMD17-01-1-0729, The Cancer Council Tasmania and The Cancer Foundation of Western Australia (www.aocstudy.org); The ANECS was supported by the National Health and Medical Research Council of Australia (339435) and The Cancer Council Tasmania. P. M. W. is supported by a fellowship from the National Health and Medical Research Council. Work on the phyto-oestrogen database was supported by research grants from the Food Standards Agency (project T05028), the Medical Research Council and Cancer Research UK. We acknowledge the contributions of Amanda Spurdle (ANECS), the AOCS Management Group (David Bowtell, Georgia Chenevix-Trench and Anna de Fazio) and the AOCS and ANECS study nurses and research assistants. The authors would like to thank Marleen Lentjes, Veronica van Scheltinga, Natasha Powell, Alison McTaggart and Amit Bhaniani for their invaluable contributions to the creation of the EPIC-Norfolk (UK) phyto-oestrogen database.

P. H. L. conceived the analysis plan and design, provided technical assistance to statistical analysis, interpreted the data and drafted the manuscript. M. C. H. contributed to the preparation of the dietary phyto-oestrogen data, carried out the statistical analysis and participated in the design of the study. T. I. I. provided assistance in the preparation of the dietary phyto-oestrogen data and writing the manuscript. A. A. M. and G. G. C. K. established the EPIC-Norfolk (UK) phyto-oestrogen database and assisted in writing the manuscript. P. M. W. has been responsible for the concept and conduct of AOCS and ANECS, and assisted in writing the manuscript. None of the authors had a conflict of interest.

\section{References}

1. Baber R (2010) Phytoestrogens and post reproductive health. Maturitas 66, 344-349.

2. Adlercreutz H (2007) Lignans and human health. Crit Rev Clin Lab Sci 44, 483-525.

3. Adlercreutz H \& Mazur W (1997) Phyto-oestrogens and Western diseases. Ann Med 29, 95-120.

4. Bhathena SJ \& Velasquez MT (2002) Beneficial role of dietary phytoestrogens in obesity and diabetes. Am J Clin Nutr 76, 11911201.

5. Tham DM, Gardner CD \& Haskell WL (1998) Clinical review 97: potential health benefits of dietary phytoestrogens: a review of the clinical, epidemiological, and mechanistic evidence. J Clin Endocrinol Metab 83, 2223-2235.

6. Adlercreutz H (2002) Phyto-oestrogens and cancer. Lancet Oncol 3, 364-373

7. Buck K, Zaineddin AK, Vrieling A, et al. (2010) Meta-analyses of lignans and enterolignans in relation to breast cancer risk. $\mathrm{Am} \mathrm{J}$ Clin Nutr 92, 141-153.

8. Hedelin M, Lof M, Olsson M, et al. (2008) Dietary phytoestrogens are not associated with risk of overall breast cancer but diets rich in coumestrol are inversely associated with risk of estrogen receptor and progesterone receptor negative breast tumors in Swedish women. J Nutr 138, 938-945.

9. Myung SK, Ju W, Choi HJ, et al. (2009) Soy intake and risk of endocrine-related gynaecological cancer: a meta-analysis. BJOG 116, 1697-1705.

10. Ward HA \& Kuhnle GG (2010) Phytoestrogen consumption and association with breast, prostate and colorectal cancer in EPIC Norfolk. Arch Biochem Biophys 501, 170-175. 
11. Ward HA, Kuhnle GG, Mulligan AA, et al. (2010) Breast, colorectal, and prostate cancer risk in the European Prospective Investigation into Cancer and Nutrition-Norfolk in relation to phytoestrogen intake derived from an improved database. Am J Clin Nutr 91, 440-448.

12. Wu AH, Yu MC, Tseng CC, et al. (2008) Epidemiology of soy exposures and breast cancer risk. Br J Cancer 98, 9-14.

13. Boker LK, Van der Schouw YT, De Kleijn MJ, et al. (2002) Intake of dietary phytoestrogens by Dutch women. J Nutr 132, 1319-1328.

14. Possemiers S, Bolca S, Eeckhaut E, et al. (2007) Metabolism of isoflavones, lignans and prenylflavonoids by intestinal bacteria: producer phenotyping and relation with intestinal community. FEMS Microbiol Ecol 61, 372-383.

15. Messina M, McCaskill-Stevens W \& Lampe JW (2006) Addressing the soy and breast cancer relationship: review, commentary, and workshop proceedings. J Natl Cancer Inst 98, 1275-1284.

16. de Kleijn MJ, van der Schouw YT, Wilson PW, et al. (2001) Intake of dietary phytoestrogens is low in postmenopausal women in the United States: the Framingham study. J Nutr 131, 1826-1832.

17. van Erp-Baart MA, Brants HA, Kiely M, et al. (2003) Isoflavone intake in four different European countries: the VENUS approach. Br J Nutr 89, Suppl. 1, S25-S30.

18. Guthrie JR, Ball M, Murkies A, et al. (2000) Dietary phytoestrogen intake in mid-life Australian-born women: relationship to health variables. Climacteric 3, 254-261.

19. Hanna KL, O’Neill S \& Lyons-Wall PM (2010) Intake of isoflavone and lignan phytoestrogens and associated demographic and lifestyle factors in older Australian women. Asia Pac J Clin Nutr 19, 540-549.

20. Merritt MA, Green AC, Nagle CM, et al. (2008) Talcum powder, chronic pelvic inflammation and NSAIDs in relation to risk of epithelial ovarian cancer. Int J Cancer 122, 170-176.

21. Rowlands IJ, Nagle CM, Spurdle AB, et al. (2011) Gynecological conditions and the risk of endometrial cancer. Gynecol Oncol 123, $537-541$.

22. Willett WC, Sampson L, Stampfer MJ, et al. (1985) Reproducibility and validity of a semiquantitative food frequency questionnaire. $\mathrm{Am}$ $J$ Epidemiol 122, 51-65.

23. Marks GC, Hughes MC \& van der Pols JC (2006) Relative validity of food intake estimates using a food frequency questionnaire is associated with sex, age, and other personal characteristics. $J$ Nutr 136, 459-465.

24. Marks GC, Hughes MC \& van der Pols JC (2006) The effect of personal characteristics on the validity of nutrient intake estimates using a food-frequency questionnaire. Public Health Nutr 9, 394-402.

25. McNaughton SA, Marks GC, Gaffney P, et al. (2005) Validation of a food-frequency questionnaire assessment of carotenoid and vitamin $\mathrm{E}$ intake using weighed food records and plasma biomarkers: the method of triads model. Eur J Clin Nutr 59, 211-218.

26. Ibiebele TI, Parekh S, Mallitt KA, et al. (2009) Reproducibility of food and nutrient intake estimates using a semi-quantitative FFQ in Australian adults. Public Health Nutr 12, 2359-2365.

27. Kuhnle GG, Dell'aquila C, Aspinall SM, et al. (2009) Phytoestrogen content of fruits and vegetables commonly consumed in the UK based on LC-MS and ${ }^{13} \mathrm{C}$-labelled standards. Food Chem 116, 542-554.

28. Kuhnle GG, Dell'aquila C, Low YL, et al. (2007) Extraction and quantification of phytoestrogens in foods using automated solidphase extraction and LC/MS/MS. Anal Chem 79, 9234-9239.

29. Kuhnle GG, Dell'Aquila C, Aspinall SM, et al. (2008) Phytoestrogen content of foods of animal origin: dairy products, eggs, meat, fish, and seafood. J Agric Food Chem 56, 10099-10104.

30. Kuhnle GGC, Dell'aquila C, Aspinall SM, et al. (2009) Phytoestrogen content of cereal and cereal-based foods consumed in the UK. Nutr Cancer 61, 302-309.
31. King RA \& Bignell CM (2000) Concentrations of isoflavone phytooestrogens and their glucosides in Australian soys beans and soya foods. Aust J Nutr Diet 57, 70-78.

32. Thompson LU, Boucher BA, Liu Z, et al. (2006) Phytoestrogen content of foods consumed in Canada, including isoflavones, lignans, and coumestan. Nutr Cancer 54, 184-201.

33. Food Standards Australia New Zealand (2006) NUTTAB 2006 Australian Food Composition Tables. Canberra: Food Standards Australia New Zealand (FNANZ).

34. Olsen CM, Bain CJ, Jordan SJ, et al. (2007) Recreational physical activity and epithelial ovarian cancer: a case-control study, systematic review, and meta-analysis. Cancer Epidemiol Biomarkers Prev 16, 2321-2330.

35. WHO (2000) Obesity: Preventing and Managing the Global Epidemic: Report of a WHO Consultation. WHO Technical Report Series (894). Geneva: World Health Organization.

36. Hedelin M, Lof M, Andersson TM, et al. (2011) Dietary phytoestrogens and the risk of ovarian cancer in the Women's Lifestyle and Health Cohort Study. Cancer Epidemiol Biomarkers Prev 20, 308-317.

37. Horn-Ross PL, John EM, Canchola AJ, et al. (2003) Phytoestrogen intake and endometrial cancer risk. J Natl Cancer Inst 95, 1158-1164.

38. Cotterchio M, Boucher BA, Kreiger N, et al. (2008) Dietary phytoestrogen intake - lignans and isoflavones - and breast cancer risk (Canada). Cancer Causes Control 19, 259-272.

39. Horn-Ross PL, Hoggatt KJ, West DW, et al. (2002) Recent diet and breast cancer risk: the California Teachers Study (USA). Cancer Causes Control 13, 407-415.

40. Touillaud MS, Thiebaut AC, Niravong M, et al. (2006) No association between dietary phytoestrogens and risk of premenopausal breast cancer in a French cohort study. Cancer Epidemiol Biomarkers Prev 15, 2574-2576.

41. Xu WH, Zheng W, Xiang YB, et al. (2004) Soya food intake and risk of endometrial cancer among Chinese women in Shanghai: population based case-control study. Br Med J 328, 1285.

42. Cassidy A, Bingham S \& Setchell K (1995) Biological effects of isoflavones in young women: importance of the chemical composition of soyabean products. Br J Nutr 74, 587-601.

43. Thompson LU, Robb P, Serraino M, et al. (1991) Mammalian lignan production from various foods. Nutr Cancer 16, 43-52.

44. Bandera EV, Williams MG, Sima C, et al. (2009) Phytoestrogen consumption and endometrial cancer risk: a population-based casecontrol study in New Jersey. Cancer Causes Control 20, 1117-1127.

45. Milder IE, Arts IC, van de Putte B, et al. (2005) Lignan contents of Dutch plant foods: a database including lariciresinol, pinoresinol, secoisolariciresinol and matairesinol. Br J Nutr 93, 393-402.

46. Keinan-Boker L, Peeters PH, Mulligan AA, et al. (2002) Soy product consumption in 10 European countries: the European Prospective Investigation into Cancer and Nutrition (EPIC) study. Public Health Nutr 5, 1217-1226.

47. Atkinson C, Skor HE, Fitzgibbons ED, et al. (2002) Overnight urinary isoflavone excretion in a population of women living in the United States, and its relationship to isoflavone intake. Cancer Epidemiol Biomarkers Prev 11, 253-260.

48. Frankenfeld CL, Patterson RE, Horner NK, et al. (2003) Validation of a soy food-frequency questionnaire and evaluation of correlates of plasma isoflavone concentrations in postmenopausal women. Am J Clin Nutr 77, 674-680.

49. Mulligan AA, Welch AA, McTaggart AA, et al. (2007) Intakes and sources of soya foods and isoflavones in a UK population cohort study (EPIC-Norfolk). Eur J Clin Nutr 61, 248-254.

50. Australian Bureau of Statistics (ABS), ABoS (2006) National Health Survey, 2004-05. Canberra: ABS.

51. Pandeya N, Williams GM, Green AC, et al. (2009) Do low control response rates always affect the findings? Assessments of smoking and obesity in two Australian case-control studies of cancer. Aust N Z J Public Health 33, 312-319. 